\title{
Clinical efficacy of eplerenone versus placebo for central serous chorioretinopathy: study protocol for the VICl randomised controlled trial
}

\author{
Abby Willcox ${ }^{1} \cdot$ Lucy Culliford $^{1} \cdot$ Lucy Ellis $^{1} \cdot$ Chris A Rogers $^{1} \cdot$ Angela Cree $^{2}{ }^{2} \cdot$ Usha Chakravarthy $^{3} \cdot$ Sarah Ennis $^{4}$. \\ Francine Behar-Cohen ${ }^{5,6,7} \cdot$ Barnaby C Reeves $\mathbb{D}^{1} \cdot$ Sobha Sivaprasad $\mathbb{D}^{8} \cdot$ Andrew Lotery $\mathbb{( D}^{2}$
}

Received: 6 April 2018 / Revised: 10 August 2018 / Accepted: 22 August 2018 / Published online: 7 September 2018

(c) The Royal College of Ophthalmologists 2018

\begin{abstract}
Aims Chronic central serous chorioretinopathy (CSCR) is poorly understood. Fluid accumulates in the subretinal space and retinal pigment epitheliopathy and neurosensory atrophy may develop. Permanent vision loss occurs in approximately one third of cases. There are no effective treatments for CSCR. Recent studies have shown the mineralocorticoid receptor antagonist, eplerenone, to be effective in resolving subretinal fluid and improving visual acuity. This trial aims to compare the safety and efficacy of eplerenone in patients with CSCR in a double-masked randomised placebo-controlled trial.

Methods Patients are randomised 1:1 to receive eplerenone with usual care or placebo with usual care for 12 months; $25 \mathrm{mg}$ per day for 1 week, then $50 \mathrm{mg}$ per day up to 12 months (unless discontinued for safety or resolution of CSCR). Key eligibility criteria are: age 18-60 years, one eye with CSCR for $\geq 4$ months duration, best-corrected visual acuity (BCVA) $>53$ and $<86$ letters and no previous treatment. The primary outcome is BCVA at 12 months. Secondary outcomes include resolution of subretinal fluid, development of macular atrophy, subfoveal choroidal thickness, changes in low luminance visual acuity, health-related quality of life and safety.

Conclusions Recruitment is complete but was slower than expected. We maintained the eligibility criteria to ensure participants had 'true' CSCR and recruited additional centres. Effective distribution of the investigational medicinal product (IMP) was achieved by implementing a database to manage ordering and accountability of IMP packs. The results will provide adequately powered evidence to inform clinical decisions about using eplerenone to treat patients with CSCR.
\end{abstract}

Electronic supplementary material The online version of this article (https://doi.org/10.1038/s41433-018-0212-2) contains supplementary material, which is available to authorised users.

\section{Sobha Sivaprasad}

Sobha.Sivaprasad@moorfields.nhs.uk

$\triangle$ Andrew Lotery

a.j.lotery@soton.ac.uk

1 Clinical Trials and Evaluation Unit, Bristol Trials Centre, Bristol Royal Infirmary, University of Bristol, Bristol BS2 8HW, UK

2 Department of Clinical and Experimental Sciences, Faculty of Medicine, University of Southampton, Southampton SO16 6YD, UK

3 Centre for Vision Sciences, The Queen's University of Belfast,

\section{Introduction}

Central serous chorioretinopathy (CSCR) is a poorly understood eye disease. Fluid that accumulates under the retina causing a neurosensory retinal detachment is a sign of pigment epitheliopathy, which can lead to permanent vision loss in up to a third of cases [1]; some resolve spontaneously but others

\section{Belfast BT12 6BA, UK}

4

Department of Human Genetics and Genomics Medicine, University of Southampton, Southampton SO16 6YD, UK

5 University of Lausanne, Lausanne, Switzerland

6 Teams 1 and 17, Centre de Recherche des Cordeliers, UMR 1138, Sorbonne Universités UPMC, Université Paris 06, Paris, France

7 Centre de Recherche des Cordeliers, UMR 1138, Sorbonne Paris Cité, Université Paris Descartes, Paris, France

8 NIHR Moorfields Biomedical Research Centre, Moorfields Eye Hospital NHS Foundation Trust, London EC1V 2PD, UK 
persist for years, recur or affect the second eye [2]. Spontaneous resolution typically occurs within 3 months of onset [2], hence persistent or recurring subretinal fluid (SRF) beyond 3 months is defined as chronic. The incidence is 10 per 100,000 men and 2 per 100,000 women [2]. The cause is unknown, although CSCR can occur in families and we recently identified the first genetic determinants [3].

There are no proven treatments and little progress has been made in understanding CSCR [2]. The current treatment of choice is photodynamic laser therapy (PDT), but there are few definitive randomised controlled trials (RCTs) supporting its use and most of the studies are small [4]. One RCT reported half-dose verteporfin PDT to have benefits in an acute CSCR population, but the effects of PDT in chronic CSCR have not been definitively studied in a placebo-controlled RCT [5]. PDT carries a risk of retinal scarring, atrophy or choroidal ischaemia. Since CSCR often resolves spontaneously [6], ophthalmologists are reluctant to use PDT. Some patients are treated with anti-vascular endothelial growth factor (anti-VEGF) therapy, but evidence to support this treatment is equivocal [7]. Most patients with chronic CSCR have no active treatment and up to a third may have permanent visual loss [1].

In a rat model of CSCR, choroidal vasodilation and SRF (a feature of CSCR) were induced by aldosterone, a mineralocorticoid receptor (MR) activator [8]. Blocking this pathway prevented choroidal thickening. Subsequently, two patients with non-resolved chronic CSCR were treated with oral eplerenone, a specific MR antagonist, for 5 weeks. Their retinal detachment and choroidal vasodilation resolved, and the associated visual acuity improvements were maintained for 5 months after stopping eplerenone [8]. These results have prompted investigation of MR blockade as a therapy to reverse CSCR.

In a subsequent small cohort of patients with chronic CSCR of at least 4 months duration, a significant reduction in central macular thickness, SRF level, and an improvement in visual acuity was observed in some patients [9]. A doublemasked RCT concluded that eplerenone was safe in patients with CSCR but was not beneficial [10], an unsurprising result given the small sample size and short-term intervention. The biology underpinning treatment with eplerenone combined with the absence of high-quality evidence provided a strong rationale to conduct a long-term double-masked RCT to test the efficacy of eplerenone in patients with chronic CSCR.

\section{Objectives}

The objectives of the VICI trial are:

(a) To evaluate whether best-corrected visual acuity (BCVA) following eplerenone treatment with usual care is superior to placebo with usual care.

(b) To evaluate resolution of SRF; safety; patient-reported visual function; the response of the choroid and retinal pigment epithelium (RPE) to treatment; low luminance visual acuity (LLVA).

(c) To generate a biobank from treatment-naive CSCR patients for future mechanistic studies.

\section{Subjects and methods}

\section{Trial design}

The VICI trial is a multicentre, individually randomised (1:1), double-masked, placebo-controlled parallel group RCT. Eligible patients who give written informed consent will be randomised to eplerenone treatment with usual care or placebo with usual care for a period of 12 months. Recruitment has taken place in 22 sites and was projected to take 12 months. Figure 1 shows the study schema.

Usual care usually comprises observation without any intervention. The protocol recommends that such treatments should only be offered if BCVA deteriorates by $\geq 15$ letters from baseline, an established criterion [11, 12]. Investigators are discouraged from offering alternative therapies; if used, information about alternative therapies is collected.

All participating sites are secondary or tertiary care NHS Trusts based in the United Kingdom. The trial has been approved by the Wales Research Ethics Committee (ref. 16/ WA/0069) and the Medicines and Healthcare products Regulatory Agency (MHRA). The principles of Good Clinical Practice will be adhered to throughout in accordance with the Declaration of Helsinki.

\section{Study population}

Inclusion criteria:

1. $\geq 18$ and $\leq 60$ years old.

2. CSCR $\geq 4$ months duration in one eye, defined as: subfoveal presence of SRF on optical coherence tomography (OCT) AND characteristic appearance of CSCR on fundus fluorescein angiogram (FFA) and indocyanine green angiography (ICGA) AND a patient history and examination consistent with CSCR having been present for $\geq 4$ months.

3. A female participant must: (a) have a negative pregnancy test and be prepared to use effective contraception during participation in the trial and 3 months after, or (b) be surgically sterile or (c) be 
Fig. 1 Trial schema showing the recruitment pathway, follow-up schedule and assessments

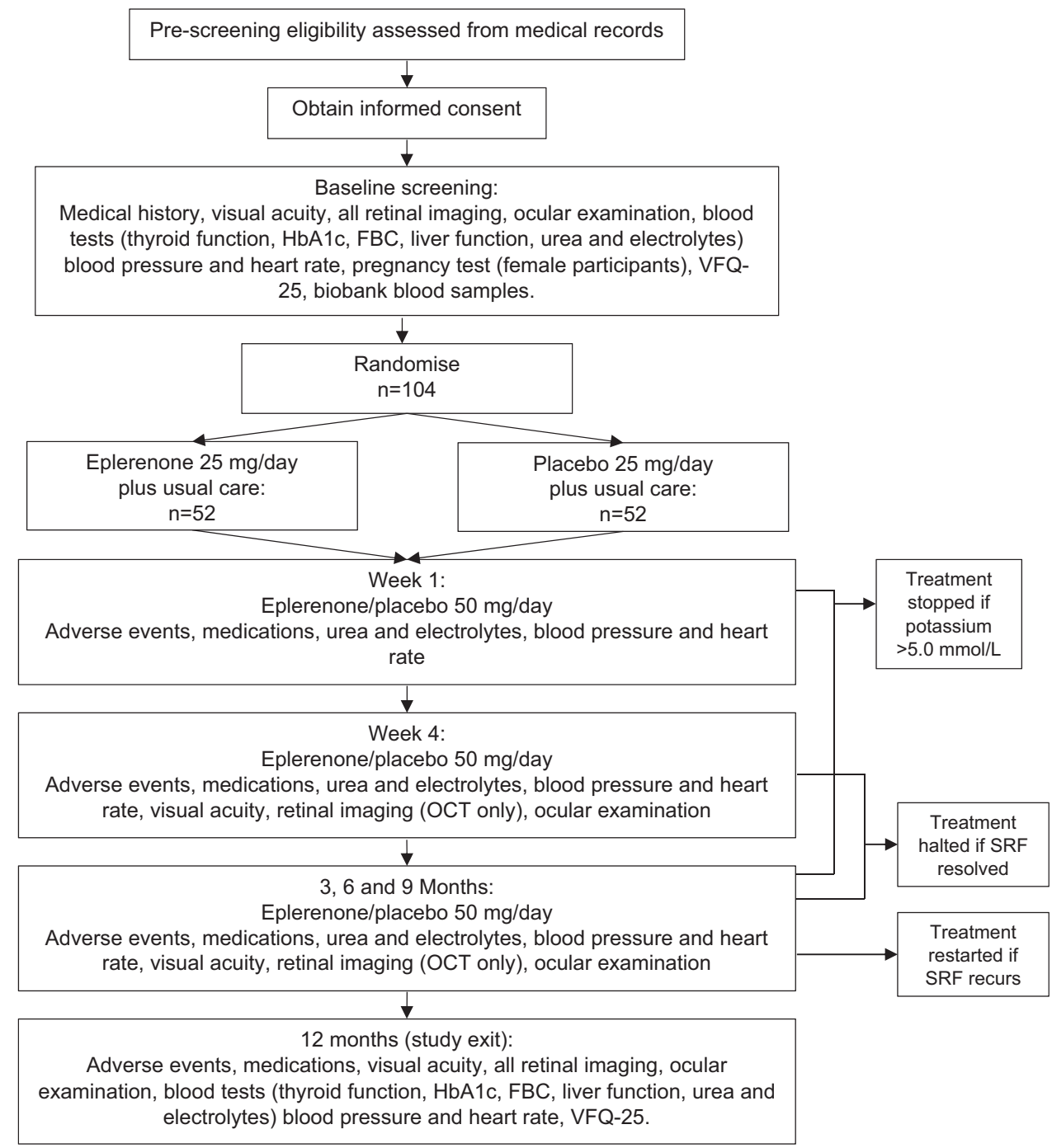

post-menopausal for $>12$ months.

4. Able to provide written informed consent.

The following inclusion criteria apply to the study eye:

5. An early treatment diabetic retinopathy study $[13,14]$, BCVA score of $>53$ and $<86$ letters.

6. Clear ocular media and adequate pupillary dilatation to permit photography.

Patient-level exclusion criteria:

1. Hyperkalaemia (serum potassium $>5.0 \mathrm{mmol} / \mathrm{L}$ ).

2. Hepatic or renal impairment (patients with severe renal insufficiency (estimated glomerular filtration rate $<30 \mathrm{~mL} / \mathrm{min}$ per $1.73 \mathrm{~m}^{2}$ ) or patients with severe hepatic insufficiency (Child-Pugh Class C; see Supplementary Information 1 for definitions).
3. Pregnancy or breast feeding.

4. Known allergy to fluorescein or indocyanine green.

5. Receiving concomitant medications (see Supplementary Information 2 for details).

6. Hypersensitivity or allergy to eplerenone or any of its excipients.

7. Hereditary galactose intolerance, Lapp lactase deficiency or glucose-galactose malabsorption.

8. Aspirin $>75 \mathrm{mg}$ per day.

The following exclusion criteria apply to the study eye:

9. Choroidal neovascularisation.

10. Previous/current treatment with eplerenone or previous/current treatment with PDT, anti-VEGF therapy, intra-ocular steroid use or thermal laser therapy for CSCR.

11. Presence of any other disease which could cause 
retinal fluid or SRF to accumulate (e.g. diabetic retinopathy, polypoidal choroidal vasculopathy, domed shaped maculopathy or choroidal haemangioma) or affect visual acuity.

12. Myopia $>6 \mathrm{D}$.

CSCR can be bilateral at presentation or may develop in the contralateral eye during the study. Treatment is given orally and any effect is through systemic absorption. Therefore, eye-specific outcomes such as BCVA and LLVA, and FA, ICGA, and OCT parameters, are measured in both eyes of participants throughout the trial.

Patients with CSCR are identified and approached according to local site procedures. All potential participants receive an invitation letter and participant information leaflet describing the study and most have $>24 \mathrm{~h}$ to consider whether to participate. A member of the local site research team answers any questions, confirms eligibility and takes written informed consent if the patient decides to participate. The principal investigator or a delegated clinician confirms eligibility before randomisation. Details of all patients approached and reason(s) for non-participation are documented.

\section{Investigational medicinal products}

The investigational medicinal product (IMP) in this trial is either (a) eplerenone (Zentiva; Guilford, UK) at $25 \mathrm{mg}$ per day, increased to $50 \mathrm{mg}$ per day after 1 week, plus usual care, or (b) placebo capsules, plus usual care. The placebo is lactose which was chosen because it is present in the licensed medication. IMP is continued until there is evidence of complete resolution of SRF or until 12 months after baseline. If SRF recurs after resolution during the follow-up period, participants re-start IMP and follow the same dose escalation procedure.

Over-encapsulated gelatin capsules mask the IMP (Newcastle Specials Pharmacy Production Unit; Newcastleupon-Tyne, UK). Capsules are packaged in plastic bottles (10 capsules of $25 \mathrm{mg}$ eplerenone/placebo per bottle; 36 capsules of $50 \mathrm{mg}$ eplerenone/placebo per bottle), distributed to sites by the manufacturing pharmacy and stored in site pharmacies at room temperature.

\section{Safety criteria and IMP cessation}

Serum potassium is measured at each follow-up time-point because hyperkalaemia is a known side effect of eplerenone. Participants switch from 25 to $50 \mathrm{mg}$ per day at week 1 , providing serum potassium is $\leq 5.0 \mathrm{mmol} / \mathrm{L}$. If serum potassium exceeds $5.0 \mathrm{mmol} / \mathrm{L}$ at any time-point, the participant stops taking the study drug and hyperkalaemia is recorded as an adverse event (AE). Such participants are invited to continue with follow-up visits up to 12 months.

\section{Safety reporting}

Data on AEs and reactions are collected throughout the follow-up period, by asking participants at each follow-up visit. The local research team also reviews a participant's medical records for hospital admissions, if a participant fails to attend. Each participant's GP is notified of their participation, with a request to inform the local research team about any suspected $\mathrm{AE}$ or reaction.

The data are recorded on case report forms. All serious AEs (SAEs) are reported to the Clinical Trials and Evaluation Unit (CTEU) Bristol within $24 \mathrm{~h}$ of the local site team becoming aware. Causality of SAEs is decided by the treating clinician. CTEU Bristol reports all SAEs to the Sponsor within $24 \mathrm{~h}$, and to the MHRA and the data monitoring and safety committee (DMSC) annually and biannually, respectively. Reporting of any suspected unexpected serious adverse reaction (SUSAR) to the MHRA, research ethics committee and DMSC is expedited (maximum of 7 days in the event of death and 15 days for all other SUSARs).

\section{Adherence to medication}

Adherence is monitored by the CTEU Bristol from data submitted by sites and reported to the trial oversight committees. The risk of non-adherence is mitigated by: regular follow-up visits (at least every 3 months) when participants are asked whether they have missed a treatment; prescribing a limited amount of IMP at each visit; requiring participants to return unused IMP capsules; recording the number of capsules returned (double-counted by nurse and pharmacist). If the observed number of capsules returned at a visit is $>5$ more than expected, the local research team is advised to maintain closer contact with the participant to encourage adherence. Reasons for non-adherence will be explored and documented.

\section{Outcomes}

\section{Primary outcome}

The primary outcome is the BCVA at the 12-month visit $[13,14]$. BCVA is assessed at baseline, 4 weeks, 3, 6, 9 and 12 months post-randomisation. 


\section{Secondary outcomes}

(a) LLVA, measured as for BCVA, immediately afterwards, by adding a 2-log neutral density filter.

(b) Central subfield retinal thickness (CSRT), measured by OCT at 12 months.

(c) SRF thickness as measured by OCT, vertically at the thickest point or sub-foveally if SRF is not thickest at the fovea.

(d) Systemic and ocular AEs at any time during follow-up.

(e) Development of macular atrophy of the RPE, defined as hypo-autofluorescence (AF) at 12 months. The area of subfoveal and total hypo-AF measured at baseline and 12 months, and atrophy assessed by measuring homogenous AF using Heidelberg Spectralis software (Franklin, MA, USA).

(f) Subfoveal choroidal thickness: One measurement at the fovea and one at the thickest macular point (in $\mu \mathrm{m}$ ), measured by enhanced depth imaging OCT at 12 months.

(g) Reduced choroidal permeability at 12 months, measured from ICGA, and graded as yes, no or cannot grade. Comparison of 12-month images to baseline will qualitatively assess changes, graded as better, worse, completely resolved or cannot grade.

(h) Time to resolution of SRF.

(i) Complete, partial (decrease in CSRT $>25 \%$ of from baseline due to resolution of SRF) or no resolution of SRF (change in CSRT $\leq \pm 25 \%$ from baseline) at each time-point of the study. Recurrence is defined as new SRF in a study eye after complete resolution of SRF.

(j) Patient-reported visual function using the Visual Functioning Questionnaire-25, version 2000 (VFQ-25) at 12 months.

(k) Classification of study eyes by each FFA phenotype, for example, smoke stack, ink-blot, chronic epitheliopathy.

(1) Classification of study eyes as early (complete or partial resolution of subfoveal SRF by 3 months from baseline), late (complete or partial resolution of subfoveal SRF after 6 months from baseline), or non-responder.

(m) Incident CSCR in the fellow eye, measured by OCT, FFA, ICGA or AF.

Heidelberg imaging equipment is mandatory to minimise inconsistency in images. Figure 2 shows the schedule of assessment of outcomes and investigations.

\section{Randomisation}

Participants are randomised within 4 weeks of the screening visit by the ophthalmologist or research nurse via a secure internet-based randomisation system (GeneSYS, CTEU Bristol, UK) [15]. Randomised allocations were generated before recruiting the first participant and supplied to the manufacturing pharmacy to label the bottles of IMP with unique bottle numbers. Allocations are concealed until a participants' identity and eligibility are captured in the trial database.

\section{Features to minimise bias}

The trial is placebo controlled. Bottles of IMP are allocated to participants by the unique bottle number. Bottles are labelled identically except for the unique number. Visual acuity examiners and imaging technicians have no information about outcomes or AEs from any previous visit when carrying out tests, minimising the risk of biasing measurements or unmasking. The interviewer who administers the VFQ-25 booklets is masked. All retinal images are graded by masked, trained and quality assured independent graders in the Network of Ophthalmic Reading Centres UK (NetWORC UK) [16]. We will report retention for each outcome, including reasons for attrition or exclusions from the analyses.

\section{IMP database}

Allocation of bottles of study drug is managed via a secure, password-protected, internet-based IMP database with site and role-restricted access. Different users (local site research teams, site pharmacists and trial management staff) access role-specific modules of the database to place orders, monitor local stock levels, etc. Further details are available in Supplementary Information 3.

\section{Unmasking}

The treating investigator can request unmasking but only in the event of a medical emergency for which knowledge of the allocation will affect the patient's care. The chief investigator or co-lead investigator has the final decision and unilateral right to unmask the allocation.

If required, unmasking can be performed by the CTEU Bristol or a local site pharmacist, using the IMP database. Local site pharmacists have sealed code-break envelopes as a back-up option in the event of internet failure, which will be collected and inspected at the end of the trial for signs of tampering. Any unmasking will be recorded and reported at the end of the trial. 
Fig. 2 SPIRIT diagram of trial time-points and data collection schedule. Trial time-points and data collection schedule.

Timepoints: $-t_{1}=$ baseline; $t_{1}$ $=$ week 1 ( \pm 1 day); $t_{2}=$ week 4 ( \pm 5 days); $t_{3}=3$ months ( \pm 10 days); $t_{4}=6$ months ( \pm 10 days); $t_{5}=9$ months ( \pm 10 days); $t_{6}=12$ months ( \pm 10 days). BCVA bestcorrected visual acuity, EDI enhanced depth imaging, NEI National Eye Institute. ${ }^{*} 1$ At each visit we will check whether patients are taking any drugs that have been shown to treat CSCR (e.g. rifampicin, finasteride, melatonin). *2 Images at baseline and 12 months to be graded by independent reading centre at Network of Ophthalmic Reading Centres UK. Images from other at time-points to be graded by specialists within the study team. *3 Where equipment is available. $* 4$ Samples sent to the University of Southampton hospital laboratory to store in the biobank. *5 Tests conducted at local hospitals. $* 6$ To include creatinine

\begin{tabular}{|c|c|c|c|c|c|c|c|c|}
\hline \multirow[b]{3}{*}{ TIMEPOINT } & \multicolumn{8}{|c|}{ STUDY PERIOD } \\
\hline & \multirow{2}{*}{$\begin{array}{c}\text { Enrolment } \\
-t_{1} \\
\end{array}$} & \multirow{2}{*}{$\frac{\text { Allocation }}{0}$} & \multicolumn{5}{|c|}{ Post-allocation } & \multirow{2}{*}{$\begin{array}{c}\text { Close-out } \\
t_{6} \\
\end{array}$} \\
\hline & & & $t_{1}$ & $t_{2}$ & $t_{3}$ & $t_{4}$ & $t_{5}$ & \\
\hline \multicolumn{9}{|l|}{ ENROLMENT: } \\
\hline \multirow{4}{*}{$\begin{array}{r}\text { pre-screen } \\
\text { Informed consent } \\
\text { Post-consent } \\
\text { eligibility screen } \\
\text { Allocation }\end{array}$} & $\mathrm{X}$ & & & & & & & \\
\hline & $x$ & & & & & & & \\
\hline & $x$ & & & & & & & \\
\hline & & $x$ & & & & & & \\
\hline \multicolumn{9}{|l|}{ INTERVENTIONS: } \\
\hline \multicolumn{9}{|l|}{ Eplerenone } \\
\hline \multicolumn{9}{|l|}{ Placebo } \\
\hline \multicolumn{9}{|l|}{ ASSESSMENTS: } \\
\hline Medical history & $x$ & & & & & & & \\
\hline \multirow{2}{*}{$\begin{array}{r}\text { Ophthalmic history } \\
\text { Concomitant } \\
\text { medications }^{* 1}\end{array}$} & $x$ & & & & & & & \\
\hline & $\mathrm{X}$ & & $x$ & $x$ & $\mathrm{x}$ & $\mathrm{X}$ & $\mathrm{x}$ & $\mathrm{X}$ \\
\hline $\begin{array}{r}\text { Pregnancy test } \\
\text { (women only) }\end{array}$ & $x$ & & & & & & & \\
\hline \multirow{2}{*}{$\begin{array}{r}\text { Fundus fluorescein } \\
\text { angiogram } \\
\text { Indocyanine green } \\
\text { angiography }\end{array}$} & $\mathrm{X}$ & & & & & & & $X$ \\
\hline & $x$ & & & & & & & $x$ \\
\hline \multirow{2}{*}{$\begin{array}{r}\text { Autofluorescence } \\
\text { Fundus } \\
\text { photography }\end{array}$} & $x$ & & & & & & & $x$ \\
\hline & $\mathrm{X}$ & & & & & & & $x$ \\
\hline $\begin{array}{r}\text { BCVA (and } \\
\text { binocular BCVA) }\end{array}$ & $\mathrm{x}$ & & & $x$ & $x$ & $\mathrm{X}$ & $x$ & $x$ \\
\hline \multirow{2}{*}{$\begin{array}{r}\text { Low Luminance } \\
\text { BCVA } \\
\text { Optical coherence } \\
\text { tomography (OCT) } \\
\text { with EDI }{ }^{*}\end{array}$} & $\mathrm{X}$ & & & $x$ & $x$ & $\mathrm{X}$ & $\mathrm{x}$ & $x$ \\
\hline & $x$ & & & $X$ & $x$ & $\mathrm{X}$ & $x$ & $X$ \\
\hline \multirow{2}{*}{$\begin{array}{r}\text { OCT angiography }{ }^{*^{3}} \\
\text { DNA, serum and } \\
\text { plasma } \star^{4}\end{array}$} & $x$ & & & & & & & $x$ \\
\hline & $x$ & & & & & & & \\
\hline \multirow{2}{*}{$\begin{array}{r}\text { HbA1 }^{*^{5}} \\
\text { Thyroid function } \\
\text { tests }^{\star^{5}}\end{array}$} & $x$ & & & & & & & $x$ \\
\hline & $x$ & & & & & & & $x$ \\
\hline Full blood count ${ }^{\star^{5}}$ & $\mathrm{x}$ & & & & & & & $x$ \\
\hline $\begin{array}{r}\text { Liver function } \\
\text { tests }{ }^{*^{5}}\end{array}$ & $\mathrm{X}$ & & & & & & & $x$ \\
\hline $\begin{array}{r}\text { Urea and } \\
\text { electrolytes }{ }^{*^{5} *^{6}}\end{array}$ & $x$ & & $x$ & $x$ & $x$ & $\mathrm{X}$ & $\mathrm{x}$ & $x$ \\
\hline Blood pressure & $x$ & & $x$ & $x$ & $x$ & $x$ & $x$ & $x$ \\
\hline \multirow{2}{*}{$\begin{array}{r}\text { Heart rate } \\
\text { Slit lamp } \\
\text { examination }\end{array}$} & $x$ & & $x$ & $x$ & $x$ & $x$ & $x$ & $x$ \\
\hline & $\mathrm{X}$ & & & $x$ & $\mathrm{x}$ & $x$ & $x$ & $x$ \\
\hline \multirow{2}{*}{$\begin{array}{r}\text { Adverse events } \\
\text { NEI Visual function } \\
\text { questionnaire-25 }\end{array}$} & $x$ & & $x$ & $x$ & $x$ & $x$ & $x$ & $x$ \\
\hline & $\mathrm{x}$ & & & & & & & $\mathrm{X}$ \\
\hline
\end{tabular}




\section{Biobank}

At baseline, eligible consented participants are asked to donate $30 \mathrm{~mL}$ of blood. Donating a blood sample is optional. Samples are sent to a biobank at the University of Southampton, UK. The blood is processed, with aliquots stored at $-80^{\circ} \mathrm{C}$ as whole blood, plasma and serum. The samples will inform future mechanistic studies about CSCR.

\section{Sample size}

A sample size of 45 patients in each group is sufficient to detect a difference of five letters in BCVA between the eplerenone and placebo groups with $90 \%$ power and $5 \%$ significance (two tailed), assuming:

(a) Standard deviation of change in BCVA is nine letters $[17,18]$.

(b) Correlation between baseline and any follow-up BCVA is 0.5 .

(c) Minimum of two follow-up assessments/participant.

(d) Correlation between BCVA on follow-up visits is 0.8 .

The target sample size is 104 , allowing for $\leq 14 \%$ dropout over the 12-month period.

\section{Plan for statistical analysis}

Outcomes measured at multiple time-points (e.g. BCVA) will be compared between study eyes in the two treatment groups using mixed models for repeated measures, adjusting for baseline, allowing all patients with data to be included in the analysis. Continuous outcomes may be transformed, if necessary. Interactions between treatment and time will be examined. If an interaction is statistically significant $(p<$ $0.05)$, changes in treatment effect with time will be reported. If an interaction is not statistically significant, an overall treatment effect will be reported. Treatment effects at 12 months will be reported with $95 \%$ confidence intervals. Cross-overs will be documented. With the exception of AEs, the analyses will be according to the intention to treat. Nonadherence to medication will also be reported; depending on the extent, the statistical analysis plan may include additional analyses to investigate the interaction between adherence and treatment. A secondary analysis will include primary outcome data from both eyes, with each eye being designated as having CSCR or not at each visit, estimating the interaction of treatment and CSCR status.

Additional analyses of the overall trial cohort will investigate associations between final visual acuity and (a) patient's age and (b) granular/confluent hypo-AF in the macula at randomisation.

No subgroup analyses are planned. However, depending on the level of adherence observed, and the availability of OCT angiography at baseline or final visit, two subgroup analyses may be described in the statistical analysis plan and carried out, testing the following interactions: (a) good/ poor adherence and treatment; (b) presence/absence of new vessels and treatment.

\section{Trial management and monitoring}

Preparation of study documents, site initiation and training, day-to-day running of the trial and monitoring of sites according to the central monitoring plan has been/is being managed by CTEU Bristol. A trial management group (TMG) (chief investigator, co-lead investigator, trial managers and key collaborators) is overseeing the trial and meets regularly to review milestones. A DMSC meets biannually to review accruing data. A trial steering committee (TSC) oversees the overall trial, receives reports and recommendations from the DMSC and TMG and has ultimate responsibility for any decision about continuation of the trial. The trial oversight committees are described in the acknowledgments section.

\section{Protocol amendments}

Version 4.0 was used when recruitment started (14 December 2016) and version 5.0 of the protocol (26 January 2017) is currently in use. The only changes between these versions were to remove fasting blood glucose from the baseline assessment and to include fundus photography at baseline and 12 months.

\section{Discussion}

Recruitment started on 14 December 2016 and ended on 28 February 2018. Recruitment has been challenging, primarily due to: (a) the detailed eligibility criteria and (b) the use of placebo as the comparator.

(a) Predominant reasons for ineligibility have been age $>60$ years or BCVA score $\geq 86$ letters. The TMG decided not to increase the upper age limit as CSCR can be difficult to diagnose and has a similar pathology to macular degeneration, which is more prevalent in older patients. Including patients in whom the underlying cause of vision loss might not be CSCR could dilute the treatment effect and risk 
harm from eplerenone treatment for no benefit. With respect to the BCVA threshold, improvement of BCVA at screening compared to presentation due to over-refraction with a plus lens has made many patients ineligible. The upper eligible BCVA score was originally 78 letters, but increased to 85 letters before the first participant was recruited. We have not increased the upper BCVA limit further because of the risk of a ceiling effect.

(b) The placebo comparator was challenging because some patients preferred to receive PDT (some participating sites are tertiary referral centres for PDT). The rarity of the condition meant that we needed to include sites that offer PDT to meet our recruitment projection.

We have encountered logistical challenges with distributing IMP bottles. Sufficient IMP bottles have been produced for 104 participants, plus a limited supply of surplus stock. Careful distribution of IMP during the recruitment phase has ensured all 22 sites are adequately stocked for both potential and randomised participants. Requiring IMP as two doses has further complicated distribution. Fewer $25 \mathrm{mg}$ bottles have been manufactured, as they are only prescribed at baseline or when restarting treatment when disease recurs, which has required frequent re-distribution of $25 \mathrm{mg}$ bottles from lower to higher recruiting sites. Overproduction could be more cost-effective than managing and redistributing the IMP stock, depending on the costs of manufacturing the IMP.

Another consideration in this trial has been the shelf-life of the IMP, which was reduced by over-encapsulation from 24 to 18 months. We manufactured the IMP ready for the original start of recruitment and delays in trial set-up resulted in IMP expiring before use, which has had cost implications. To optimise the management and accountability of the IMP, we designed an internet-based, rolerestricted, IMP management database (Supplementary Information 3).

Monitoring adherence to the intervention is an important consideration for the management of this trial as participants are responsible for administering the IMP at home. The impact of non-adherence on the trial is twofold: dilution of the treatment effect; potential to undermine safety monitoring processes (e.g. advice to continue taking the IMP based on serum potassium results). Adherence to the intervention is closely monitored as described in the Subjects and methods section. The trial is relatively low risk with regards to the safety considerations of administering the IMP at home (e.g. overdosing). Eplerenone has a short half-life, low toxicity and is non-addictive. There are no known cases of overdosing from eplerenone and the most likely manifestations of an overdose are anticipated to be hyperkalaemia or hypotension, clinical indicators of which are being monitored at follow-up visits, with participants being withdrawn if necessary.

The results of this trial will fill a gap in the knowledge regarding the efficacy and safety of eplerenone for the treatment of CSCR in the longer term. This will include data on the rate of disease resolution and subsequent recurrence with eplerenone, something which is as yet unknown in this population. As patients with CSCR have limited therapeutic options, further evidence on the actions of eplerenone treatment for CSCR would be welcomed and could help inform future treatment decisions.

Data collection for this trial is ongoing. After publication of the trial results, the anonymised data will be made available upon reasonable request to the Sponsor institution, University Hospital Southampton NHS Foundation Trust. A statement on data sharing is included in the protocol [19].

\section{Summary}

\section{What was known before}

- CSCR is a poorly understood eye disease.

- Fluid gathers under the retina causing a neurosensory retinal detachment which can lead to permanent vision loss in up to a third of cases

- There are no proven treatments and little progress has been made in understanding CSCR.

- Improvements in visual acuity have been shown following treatment with MR antagonists.

\section{What this study adds}

- The VICI trial is the first long-term, adequately powered double-masked RCT investigating the safety and efficacy of eplerenone in CSCR.

- Participants will receive eplerenone plus usual care or placebo plus usual care for up to 12 months.

- The primary outcome is BCVA at 12 months.

- Secondary outcomes include resolution of SRF, development of macular atrophy, subfoveal choroidal thickness, changes in LLVA, health-related quality of life and safety.

Acknowledgements We would like to acknowledge the Macular Society and Fight for Sight for their feedback on the patient information leaflet and the DMSC and TSC for their oversight of the trial. The independent DMSC is formed of a Chairperson, two consultant ophthalmologists and one consultant cardiologist. The independent TSC is formed of a Chairperson, two consultant ophthalmologists, a consultant cardiologist, an ophthalmic statistician and patient and public involvement representatives; other TSC members with observer status represent the trial management team and the Sponsor. 
Funding This project is funded by the Efficacy and Mechanism Evaluation (EME) Programme, an MRC and NIHR partnership (ref: 13/94/15), with contributions from the CSO in Scotland and NISCHR in Wales and the HSC R\&D Division, Public Health Agency in Northern Ireland. The trial is sponsored by University Hospital Southampton NHS Foundation Trust, Southampton, UK. This study was designed and delivered in collaboration with the Clinical Trials and Evaluation Unit (CTEU), a UKCRC registered clinical trials unit which, as part of the Bristol Trials Centre, is in receipt of National Institute for Health Research CTU support funding.

Author contributions AL conceived the trial; AL, SS, BCR, AC and CR obtained funding; AL, SS, BCR and CR designed the trial; AW, $\mathrm{LE}$ and LC managed the trial with input from AL, SS, BCR and CR; $\mathrm{UC}, \mathrm{SE}$ and FB-C provided expert input; $\mathrm{AC}$ and $\mathrm{AL}$ set up the biobank; AW wrote the first draft of the manuscript. All authors reviewed the manuscript and amended/approved the final version

\section{Compliance with ethical standards}

Conflict of interest AW, LC, LE, CAR, AC, SE and BCR have no conflicts of interest. FB-C is an inventor on a patent protecting the use of MR antagonists in CSCR. SS has received research grants, travel grants, and speaker fees from Novartis, Bayer, Allergan, Roche, Heidelberg Engineering, and Optos. AL has received travel grants and speaker fees from Bayer and Roche.

Disclaimer The views expressed in this publication are those of the author(s) and not necessarily those of the MRC, NHS, NIHR or the Department of Health.

\section{References}

1. Loo RH, Scott IU, Flynn HW Jr, Gass JD, Murray TG, Lewis ML, et al. Factors associated with reduced visual acuity during longterm follow-up of patients with idiopathic central serous chorioretinopathy. Retina. 2002;22:19-24. PubMed PMID: 11884873.

2. Gemenetzi M, De Salvo G, Lotery AJ. Central serous chorioretinopathy: an update on pathogenesis and treatment. Eye (Lond). 2010;24:1743-56. https://doi.org/10.1038/eye.2010.130. PubMed PMID: 20930852.

3. Schubert C, Pryds A, Zeng S, Xie Y, Freund KB, Spaide RF, et al. Cadherin 5 is regulated by corticosteroids and associated with central serous chorioretinopathy. Hum Mutat. 2014;35:859-67. https://doi.org/10.1002/humu.22551. PubMed PMID: 24665005; PubMed Central PMCID: PMCPMC4215937.

4. Erikitola OC, Crosby-Nwaobi R, Lotery AJ, Sivaprasad S. Photodynamic therapy for central serous chorioretinopathy. Eye (Lond). 2014;28:944-57. Epub 2014/06/21. https://doi.org/10. 1038/eye.2014.134. PubMed PMID: 24946843; PubMed Central PMCID: PMCPMC4135258.

5. Chan WM, Lai TY, Lai RY, Liu DT, Lam DS. Half-dose verteporfin photodynamic therapy for acute central serous chorioretinopathy: one-year results of a randomized controlled trial. Ophthalmology. 2008;115:1756-65. https://doi.org/10.1016/j. ophtha.2008.04.014. PubMed PMID: 18538401.
6. Salehi M, Wenick AS, Law HA, Evans JR, Gehlbach P. Interventions for central serous chorioretinopathy: a network meta-analysis. Cochrane Database Syst Rev. 2015;12:CD011841. https://doi.org/ 10.1002/14651858.CD011841.pub2. PubMed PMID: 26691378.

7. Chung YR, Seo EJ, Lew HM, Lee KH. Lack of positive effect of intravitreal bevacizumab in central serous chorioretinopathy: meta-analysis and review. Eye (Lond). 2013;27:1339-46. Epub 2013/11/10. doi: 10.1038/eye.2013.236. PubMed PMID: 24202051; PubMed Central PMCID: PMCPMC3869506.

8. Zhao M, Celerier I, Bousquet E, Jeanny JC, Jonet L, Savoldelli M, et al. Mineralocorticoid receptor is involved in rat and human ocular chorioretinopathy. J Clin Invest. 2012;122:2672-9. https://doi.org/ 10.1172/Jci61427. PubMed PMID: WOS:000306044600037.

9. Bousquet E, Beydoun T, Zhao M, Hassan L, Offret O, Behar-Cohen F. Mineralocorticoid receptor antagonism in the treatment of chronic central serous chorioretinopathy: a pilot study. Retina-J Ret Vit Dis. 2013;33:2096-102. PubMed PMID: WOS:000330237900014.

10. Schwartz R, Habot-Wilner Z, Martinez MR, Nutman A, Goldenberg D, Cohen S, et al. Eplerenone for chronic central serous chorioretinopathy - a randomized controlled prospective study. Acta Ophthalmol. 2017. Epub 2017/06/28. https://doi.org/10. 1111/aos.13491. PubMed PMID: 28653813.

11. Brown DM, Michels M, Kaiser PK, Heier JS, Sy JP, Ianchulev T, et al. Ranibizumab versus verteporfin photodynamic therapy for neovascular age-related macular degeneration: Two-year results of the ANCHOR study. Ophthalmology. 2009;116:57-65e5. https://doi.org/10.1016/j.ophtha.2008.10.018. PubMed PMID: 19118696.

12. Rosenfeld PJ, Brown DM, Heier JS, Boyer DS, Kaiser PK, Chung $\mathrm{CY}$, et al. Ranibizumab for neovascular age-related macular degeneration. N Engl J Med. 2006;355:1419-31. https://doi.org/ 10.1056/NEJMoa054481. PubMed PMID: 17021318.

13. Beck RW, Maguire MG, Bressler NM, Glassman AR, Lindblad AS, Ferris FL. Visual acuity as an outcome measure in clinical trials of retinal diseases. Ophthalmology. 2007;114:1804-9. https://doi.org/10.1016/j.ophtha.2007.06.047. Epub 2007/10/ 03PubMed PMID: 17908590.

14. Ferris FL III, Kassoff A, Bresnick GH, Bailey I. New visual acuity charts for clinical research. Am J Ophthalmol. 1982;94:91-6. Epub 1982/07/01. PubMed PMID: 7091289.

15. Hutton D, Smith N, Cappel-Porter H, Saw C, Rogers CA. Development of the "GeneSYS" database system to support trial data capture and conduct. 2013. In: Trials [Internet]. 29/11/ 2013. [66].

16. NetWORC UK. Network of ophthalmic reading centres UK. Available from: http://www.networcuk.com/.

17. Reeves BC, Wood JM, Hill AR. Reliability of high- and lowcontrast letter charts. Ophthalmic Physiol Opt. 1993;13:17-26. Epub 1993/01/01. PubMed PMID: 8510943.

18. Becker R, Teichler G, Graf M. Reproducibility of visual acuity assessment in normal and low visual acuity. Strabismus. 2007;15:3-6. https://doi.org/10.1080/09273970601172435. Epub 2007/05/25PubMed PMID: 17523039.

19. Clinical efficacy and mechanistic evaluation of Eplerenone for central serous chorio-retinopathy the VICI study. 2016. Available from: https://njl-admin.nihr.ac.uk/document/download/ 2009989. 